\title{
Optical Leaky Wave Antenna Experiment Demonstration and Electronic Modulation Investigation
}

\author{
Qiancheng Zhao, Yuewang Huang, Caner Guclu, Filippo Capolino and Ozdal Boyraz \\ Electrical Engineering and Computer Science, University of California, Irvine, Irvine, California, USA, 92697 \\ oboyraz@uci.edu
}

\begin{abstract}
Fabrication and characterization of optical leaky wave antennas with dielectric waveguides and semiconductor perturbations is presented. Directive radiation at $1550 \mathrm{~nm}$ is measured. Detailed study on electrical modulation capability with bandwidth exceeding $75 \mathrm{GHz}$ is presented.
\end{abstract}

\section{Introduction}

Optical leaky wave antennas (OLWAs) with tunable radiation pattern are attractive as integrated optical modulation and scanning devices [1]. Up to date, several devices have been demonstrated based on resonator structure [2], plasmonic structure [3] and etc. In this work, we experimentally demonstrate fabrication and characterization results of CMOS compatible OLWA composed of silicon nitride waveguides and silicon wire perturbations, which are designed for possible optical [4] and electronic control . The measured far-field pattern exhibits dominant peak around $80^{\circ}$. Radiation peak shifts $6 \mu \mathrm{m}$ when switching wavelength from $1540 \mathrm{~nm}$ to $1560 \mathrm{~nm}$, proving the wavelength dependent radiation angle. Investigation on the electronic modulation on far-field radiation pattern via plasma dispersion effect and Franz-Keldysh effect is also discussed. The electrical modulation bandwidth can be 75 $\mathrm{GHz}$ in carrier depletion mode, while the refractive index change can reach 0.01 using Franz-Keldysh effect. The antenna device is promising in space-multiplexing, planar imaging and etc.

\section{Fabrication and experiment characterizations}

The fabrication of the OLWA starts with SOI wafer. The device layer was thinned to $150 \mathrm{~nm}$ to achieve silicon perturbations with desired thickness. The patterned silicon device layer was then RIE etched. The waveguide was made of stoichiometric silicon nitride deposited by low-pressure chemical vapor deposition (LPCVD). A two-cycle deposition process was employed to achieve $735 \mathrm{~nm}$ thick film, and each cycle was followed by 4 hours annealing at $1100{ }^{\circ} \mathrm{C}$. The waveguide was fabricated by standard lithography process and isotropic oxygen plasma etching to improve waveguide quality. Figure 1 shows the schematic of the design and the SEM picture of the fabricated device. Final device has a $1 \mu \mathrm{m}$ wide and $735 \mathrm{~nm}$ high SiN waveguide sitting on 50 silicon perturbations with $1 \mu \mathrm{m}$ spatial periodicity. The height of the silicon perturbations is $h=150 \mathrm{~nm}$ and the width is $l=260 \mathrm{~nm}$.

(a)

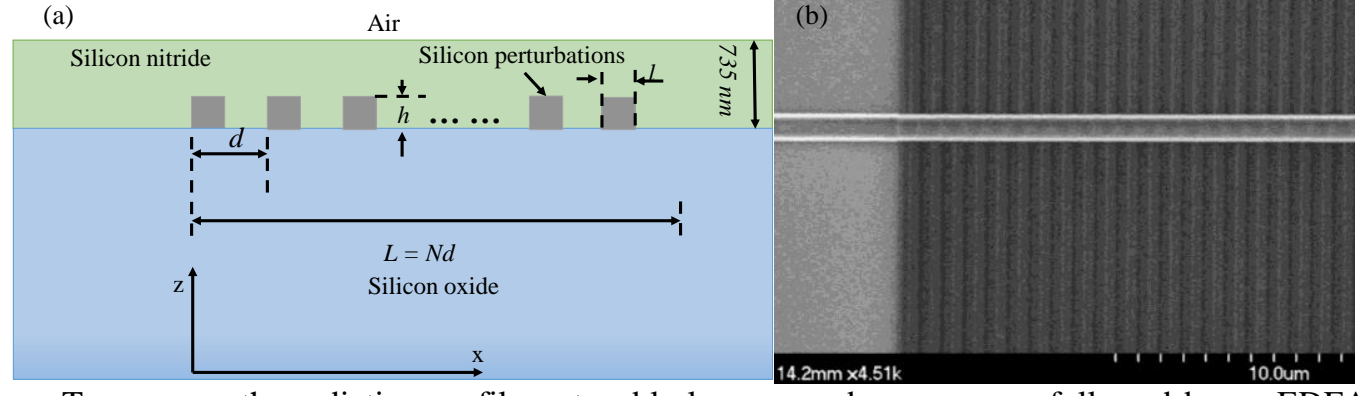

Figure 1 (a) Schematic of the OLWA with structure parameters. (b) SEM picture of the fabricated waveguide sitting on the silicon perturbations.

To measure the radiation profile, a tunable laser served as a source, followed by an EDFA and a polarization controller, butt-coupling light into the waveguide with the help of a tapered lens fiber. A cleaved single mode (SM) fiber tip, mounted on a precision stage vertically, was employed to collect radiation power. The fiber tip angle and radiation angle are characterized by $\beta$ and $\alpha$ respectively. We swept the fiber tip along the waveguide and mapped the power profile to horizontal position as shown in Figure 2 (a). Directive radiation at $1540 \mathrm{~nm}$ was observed, and radiation power was best coupled when fiber tip was tilted around $10^{\circ}$, which means radiation angle was $80^{\circ}$ (Figure 2 (b)). The result is close to our expectations [1]. The radiation profile was also measured with three different wavelengths. As illustrated in Figure 2 (c), the peak location at $1540 \mathrm{~nm}$ and $1560 \mathrm{~nm}$ was shifted by $6 \mu \mathrm{m}$, which proved that the radiation angle was wavelength dependent. All the measurements started at the same point and maintained the same fiber tip vertical distance for fair comparison. We also tested the device with different fiber tip angles and orthogonal polarization states to avoid spurious peaks. 

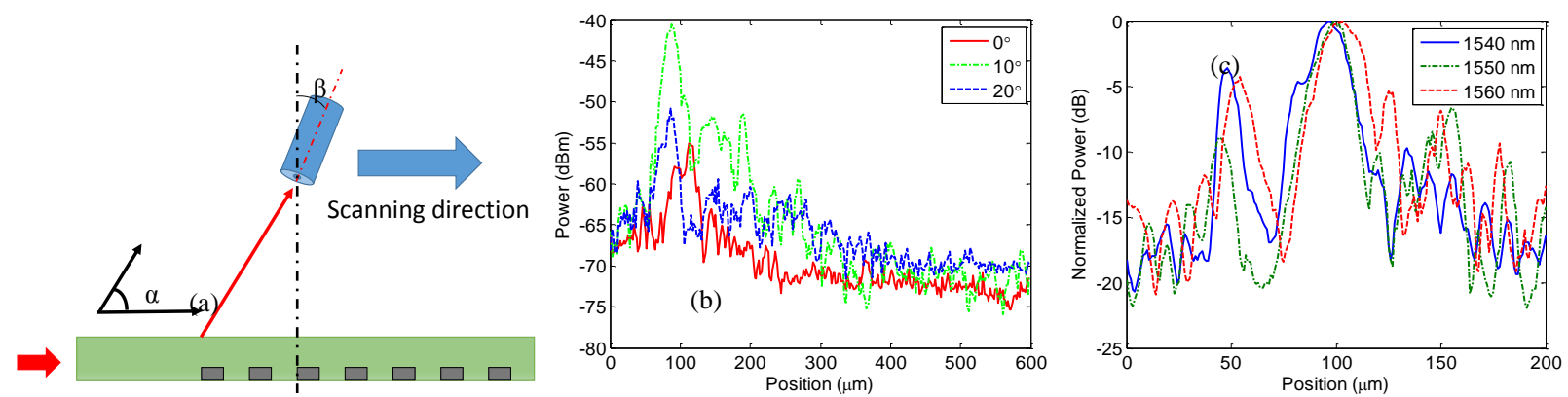

Figure 2 (a) Schematic of the fiber tip and waveguide configurations. The red thick arrow indicates the wave propagation direction. The red thin arrow sketches the possible radiation angle $\alpha$. The fiber tip moved along the wave propagation direction. The tiled angle between fiber tip and waveguide surface normal is symbolized by $\beta$. (b) Radiation profile comparison when the fiber tip was titled $0^{\circ}, 10^{\circ}$ and $20^{\circ}$. (c) Normalized radiation profile at $1540 \mathrm{~nm}, 1550 \mathrm{~nm}$, and $1560 \mathrm{~nm}$ wavelengths when the fiber tip is tilted $0^{\circ}$.

\section{Electronic modulation on far-field radiation pattern}

The use of silicon wire as perturbations facilitate electronic modulation of radiation. Here, we investigate modulating silicon refractive index by carrier depletion method and Franz-Keldysh effect [5]. In order to asses the modulation capabilities of OLWAs, $2 \mu \mathrm{m}$ long silicon wire perturbations were used in p-i-n diode configurations. The two ends are heavily doped to $10^{19} \mathrm{~cm}^{-3}$ for good ohmic contact with aluminum nodes. The $\mathrm{n}++$ region has an optimum length of $0.8 \mu \mathrm{m}$, while the $\mathrm{p}++$ region is $0.5 \mu \mathrm{m}$ long for the sake of symmetric space charge region. Ptype silicon is preferred in the intrinsic part due to higher impact on refractive index. A moderate doping level of $10^{17} \mathrm{~cm}^{-3}$ is found to be optimum, and the theoretical refractive index change is $3.4 \times 10^{-4}$ using Drude's model when applied $5 \mathrm{~V}$ reverse bias. The boundary of the depletion region is defined where the carrier density drops to 1/10 of the doping concentration, and the modulation width is defined as the difference between maximum and minimum depletion widths. The modulation width ac response was also investigated to explore device bandwidth. Modulation width was normalized to DC bias condition for a fair comparison. It can be concluded that higher doping concentration has a higher bandwidth. For $\mathrm{N}_{\mathrm{a}}=1 \times 10^{17} \mathrm{~cm}^{-3}$, its $-3 \mathrm{~dB}$ bandwidth is $75 \mathrm{GHz}$.
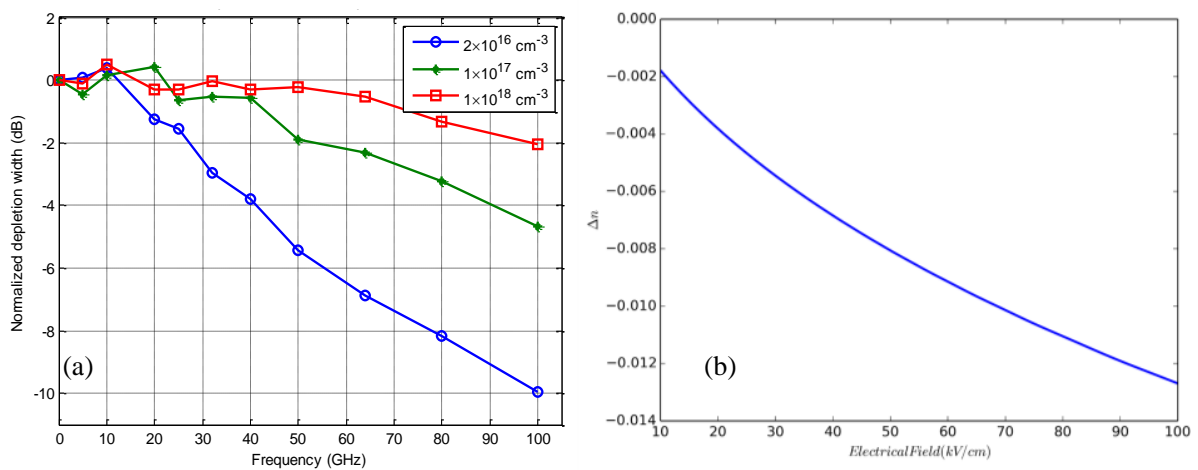

(c) Al contact n++ $\quad p+\quad p++$ Al contact

Figure 3 (a) AC response of normalized modulation width with 5 $\mathrm{V}_{\mathrm{pp}}$ square wave input. (b) Refractive index change in a germanium diode by Franz-Keldysh effect as a function of electrical field. (c) The doping profile of the silicon perturbations for carrier depletion mode.

Carrier depletion mode is limited by refractive index variation, so Franz-Keldysh effect on germanium is explored as an alternative approach. By varying the electric field in a germanium wire diode, absorption coefficient is affected, and the refractive index is altered according to Kramers-Kronig relations, as shown in Figure 3(b). The refractive index change can be as large as 0.01 near $1550 \mathrm{~nm}$ wavelength, when the applied electric field is 70 $\mathrm{kV} / \mathrm{cm}$. The modulation bandwidth is estimated to exceed far beyond $\mathrm{p}-\mathrm{i}-\mathrm{n}$ diode configuration.

\section{Conclusions and acknowledgement}

As a summary, we fabricated and tested the OLWA device. Electronic modulation by carrier depletion and FranzKeldysh effect are investigated.

This work is supported by the National Science Foundation Award \# ECCS-1028727.

\section{References}

[1]Q. Song, et.al "Silicon-based optical leaky wave antenna with narrow beam radiation," Opt. Express, vol. 19, no. 9, pp. 8735-8749, Apr. 2011. [2]S. Campione, et.al, "An optical leaky wave antenna with Si perturbations inside a resonator for enhanced optical control of the radiation," Opt. Express, vol. 20, no. 19, pp. 21305-21317, Sep. 2012.

[3]D. R. Jackson, et.al, "The role of leaky plasmon waves in thedirective beaming of light through asubwavelength aperture," Opt. Express, vol. 16, no. 26, pp. 21271-21281, Dec. 2008.

[4]S. Campione, et.al, "An optical leaky wave antenna with silicon perturbations for electronic control," 2011, p. 81200G-81200G-11.

[5]G. T. Reed, et.al, “Silicon optical modulators,” Nat. Photonics, vol. 4, no. 8, pp. 518-526, Aug. 2010. 